\section{Eye movement desensitization and reprocessing therapy for personality disorders in older adults?}

Eye Movement Desensitization and Reprocessing (EMDR) is a kind of psychotherapy, which is growing in popularity, particularly for treatment of post-traumatic stress disorder (PTSD). When Shapiro first introduced EMDR in 1989, it was approached as a controversial treatment because of lack of evidence. However, nowadays there is growing evidence for EMDR efficacy in PTSD (Mc Guire et al., 2014) and EMDR is recommended by international and national treatment guidelines for PTSD. Moreover, EMDR is also used for the treatment of other anxiety disorders, such as panic disorders (De Jongh et al., 2002). Furthermore, research continues on effects of EMDR in addiction, somatoform disorders and psychosis. So far, there is no empirical research on the efficacy of EMDR treatment in older adults.

EMDR seems to have a direct effect on restoring the distressing memory. The idea is that the memory and associated stimuli of the event are inadequately processed, and are stored in a dysfunctional manner. The goal of EMDR therapy is to process these distressing memories, reducing their lingering influence, and allowing patients to develop more adaptive coping mechanisms. During the processing phases of EMDR, the patient focuses on disturbing memory in multiple brief sets of about 15-30 seconds. Simultaneously, the patient focuses on the dual attention stimulus (e.g. therapistdirected lateral eye movement, alternate handtapping, or bilateral auditory tones). This process of alternating dual attention and personal association is repeated many times during the session. The sessions are subdivided to address the past, present, and future aspects of a traumatic or distressing memory that has been dysfunctional stored. Most of the EMDR sessions take 60 to 90 minutes with a frequency of sessions between 5 and 15 usually on a weekly basis. However, for patients with a history of multiple traumatic experiences mostly more EMDR sessions are needed.

The positive effects of EMDR on main dysfunctional cognitions have also been described in personality disorders (PDs). People with PDs act in dysfunctional ways because their core beliefs about themselves, others and the world lead to misinterpretation of situations. For example, the core belief "you can't trust anybody" in a borderline PD leads a person to become vigilant for rejection. This is comparable with complex PTSD in which disturbing core beliefs arise from traumatic experiences and these disturbing beliefs result in interpersonal problems. When using EMDR in complex PTSD, detection (and reprocessing) of memories of etiological experiences happens through main dysfunctional cognitions and these key memories will be desensitized. For example, someone has to cope with the aftermath of being bullied as a child which makes him feel like a stupid person. As a consequence, the main dysfunctional cognition "I'm worthless" has arisen and leads to symptoms of social withdrawal, anxiety to start something new, and feelings of depression. If the main dysfunctional cognition "I'm worthless" loses strength, then our hypothesis is that symptoms may reduce because new experiences will be gained. Therefore, EMDR may also be a suitable treatment for PDs in older adults. So far, little is known about the treatment of PDs in older adults. Videler et al. (2014) found fair outcomes of schema therapy for PDs in Dutch elderly outpatients. Schema therapy improves dysfunctional schemas into adaptive schemas by techniques such as "imagery rescripting;" the patient imagines the original traumatic event and creates a new script with a more satisfactory outcome.

In EMDR "imagery rescripting" becomes a form of "cognitive interweave". A cognitive interweave introduces new information or a new perspective into the processing system to proceed with the process. In PDs it's often necessary to use cognitive interweaves for successful processing painful experiences. However, empirical research of EMDR in older adults with PDs is missing. The main benefit of EMDR, compared to more comprehensive forms of psychotherapy such as schema therapy, is that EMDR sessions work fast in processing deep-seated painful experiences. Besides, EMDR may be less intensive because processing happens without discussing upsetting experiences in great detail. Neither there are homework assignments. Intellectual and cognitive function is not important for effective EMDR therapy, based on positive treatment outcome in children and people with an intellectual disability (Mevissen et al., 2012; Diehle et al., 2015). Concerning schema therapy, however, patients are expected to have good intellectual and cognitive capacities.

In short: EMDR is a relatively straightforward treatment method, easy to use, also when cognitive functioning is suboptimal with relatively modest frequency of sessions compared to other psychotherapeutic interventions such as cognitive behavioral therapy or schema therapy. Therefore, we suggest EMDR is especially suitable for older adults. As far as we know, EMDR efficacy research 
on both adults and older adults with PDs is lacking. A proof of concept study to investigate the treatment effect of EMDR in older adults with PDs is a first step to explore the added value of EMDR in this population.

\section{Conflict of interest}

None.

\section{References}

De Jongh, A., Van den Oord, H.J.M. and Ten Broeke, E. (2002). Efficacy of eye movement desensitization and reprocessing (EMDR) in the treatment of specific phobias: four single-case studies on dental phobia. Fournal of Clinical Psychology, 58, 1489-1503.

Diehle, J., Opmeer, B. C., Boer, F., Mannarino, A. p. and Lindauer, R. J. (2015). Trauma-focused cognitive behavioral therapy or eye movement desentization and reprocessing: what works in children with posttraumatic

doi:10.1017/\$1041610216000879

\section{Where is the happiness in dementia?}

Our current research aims to explore how the emotional experiences of individuals with dementia are understood, and to improve the design and delivery of care interventions. A preliminary, incidental, finding from our initial systematic literature search is reported here. Increasingly, the experience of dementia is understood from the viewpoint of the individual. However, this is not reflected in the body of research literature, which is predominantly orientated towards detailing the neuropsychiatric symptoms of mood, cognition, behavior, or physiology and "managing" the condition (Cerejeira et al., 2012). In this way, the whole and varied experiences of dementia are not recognized. There is a distance between these differing perspectives of dementia in the available literature.

Twelve electronic databases were searched between January and April 2016. The systematic review was a means of answering the question: how is emotional distress characterized for individuals with dementia? The databases searched were Applied Social Sciences Index and Abstracts (ASSIA), British Nursing Index (BNI), Cochrane Library, Cumulative Index of Nursing and Allied Health Literature (CINAHL), Health Management Information Consortium (HMIC), International stress symptoms? A randomized controlled trial. European Child \& Adolescent Psychiatry, 24, 227-236.

Mc Guire, T. M., Lee, C. W. and Drommond, P. D. (2014). Potential of eye movement desentisitation reprocessing therapy in the treatment of post-traumatic stress disorder. Fournal of Psychology Research and Behaviour Management, 26, 273-283.

Mevissen, L., Lievegoed, R., Seubert, A. and De Jongh, A. (2012). Treatment of PTSD in people with severe intellectual disabilities: a case series. Developmental Neurohabilitation, 15, 223-232.

Videler, A., Rossi, G. M. P., Schoevaars, M., Van der Feltz, C. and Van Alphen, S. P. J. (2014). Effects of schema group therapy in older outpatients: a proof of concept study. International Psychogeriatrics, 26, 1-9.

E. M. J. Gielkens, ${ }^{1}$ S. SobCZAK ${ }^{1,2}$ AND S. P. J. VAN Alphen ${ }^{1,3}$

${ }^{1}$ Department of Old Age Psychiatry, Mondriaan Hospital, Heerlen-Maastricht, the Netherlands ${ }^{2}$ Department of Psychiatry and Neuropsychology, Maastricht University, Maastricht, the Netherlands ${ }^{3}$ Department of Clinical and Life Span Psychology, Vrije Universiteit Brussel (VUB), Brussels, Belgium Email: b.van.alphen@mondriaan.eu

Bibliography of the Social Sciences (IBSS), Medline, ProQuest Dissertation \& Theses Global (PQDT), Psychological Information Database (PsycINFO), Scopus, The Campbell Library and Web of Science, offering an international scope of the published and grey literature. Our search was limited to papers in English which may cause some bias; in addition the selected databases include some further bias. A search strategy was devised in attempt to capture all the relevant literature (Petticrew and Roberts, 2006). Terms were informed by Medical Subject headings (MeSH), subject headings/thesaurus terms for each database and keywords, using truncation and wildcards to accommodate different word endings and spellings. Search terms were "dementia" or "Alzheimer" and as many emotion synonyms as we could identify from subject headings/thesaurus terms and preliminary reading of relevant literature: emotion, distress, affect, feeling, depression, anxiety, anger, shame, fear, well-being, sadness, agitation, apathy, grief, jealousy, hopelessness, loneliness, jealousy, happiness, and desire. "Happiness" and "desire" were the only thesaurus terms that were available to maximize the retrieval of "positive emotion" relevant titles.

In the process of completing this comprehensive literature search, it became apparent that a surprisingly small proportion of the literature concerned with the emotional experience of individuals with dementia was concerned with positive emotional experiences. Of the approximately 40,000 titles 